\title{
THE SWALLOWTAIL INTEGRAL IN THE HIGHLY OSCILLATORY REGION II*
}

\author{
CHELO FERREIRA ${ }^{\dagger}$, JOSÉ L. LÓPEZ ${ }^{\ddagger}$, AND ESTER PÉREZ SINUSíA ${ }^{\dagger}$
}

\begin{abstract}
We analyze the asymptotic behavior of the swallowtail integral $\int_{-\infty}^{\infty} e^{i\left(t^{5}+x t^{3}+y t^{2}+z t\right)} d t$ for large values of $|y|$ and bounded values of $|x|$ and $|z|$. We use the simplified saddle point method introduced in [López et al., J. Math. Anal. Appl., 354 (2009), pp. 347-359]. With this method, the analysis is more straightforward than with the standard saddle point method, and it is possible to derive complete asymptotic expansions of the integral for large $|y|$ and fixed $x$ and $z$. There are four Stokes lines in the sector $(-\pi, \pi]$ that divide the complex $y$-plane into four sectors in which the swallowtail integral behaves differently when $|y|$ is large. The asymptotic approximation is the sum of two asymptotic series whose terms are elementary functions of $x, y$, and $z$. One of them is of Poincaré type and is given in terms of inverse powers of $y^{1 / 2}$. The other one is given in terms of an asymptotic sequence whose terms are of the order of inverse powers of $y^{1 / 9}$ when $|y| \rightarrow \infty$, and it is multiplied by an exponential factor that behaves differently in the four mentioned sectors. Some numerical experiments illustrate the accuracy of the approximation.
\end{abstract}

Key words. swallowtail integral, asymptotic expansions, modified saddle point method

AMS subject classifications. 33E20, 41A60

1. Introduction. The swallowtail integral is one of the most important canonical diffraction integrals [1, Chap. 36], which are integrals that are an essential ingredient in the modelling of many physical phenomena, especially those related to wave propagation; see $[3,2,6,12,14,16,17]$ or the references therein for detailed information. Apart from their physical applications, the canonical diffraction integrals have an important mathematical application in the uniform asymptotic approximation of oscillatory integrals [13]. For a comprehensive description of these integrals (symmetries, illustrative pictures, scaling relations, convergent series expansions, zeros, differential equations, bifurcation sets,...), see [1, Chap. 36].

The canonical diffraction integrals are classified according to the number of free independent parameters that describe the type of singularities arising in catastrophe theory. The simplest integral in this hierarchy is the well-known integral representation of the Airy function, and the second one is the Pearcey integral. In this paper we are concerned with the third one: the swallowtail catastrophe. The canonical form of the oscillatory integral describing the swallowtail diffraction catastrophe is given by the swallowtail catastrophe integral [1, Chap. 36, Sec. 2, eq. 36.2.4]:

$$
\Psi(x, y, z):=\int_{-\infty}^{\infty} e^{i\left(t^{5}+x t^{3}+y t^{2}+z t\right)} d t
$$

The computation of this integral is not straightforward because of the oscillatory character of the integrand. Some numerical methods based on the numerical integration of certain differential equations satisfied by the swallowtail integral or on complex contour quadrature techniques may be found in [4] and [5]. A convergent expansion in terms of powers of $x, y$, and $z$ is given in [1, Sec. 36, Chap. 8, eq. 36.8.1], but the convergence speed of this expansion is rather slow for moderate or large values of the variables. On the other hand, asymptotic expansions of this integral are not fully investigated. In [8] we can find some information about the asymptotics of $\Psi(x, y, z)$, but a complete asymptotic expansion is not given.

\footnotetext{
*Received March 8, 2019. Accepted December 13, 2019. Published online on February 4, 2020. Recommended by F. Marcellan.

${ }^{\dagger}$ Dpto. de Matemática Aplicada, IUMA, Universidad de Zaragoza, Zaragoza ( $\{$ cferrei, ester.perez\} @unizar.es).

${ }^{\ddagger}$ Dpto. de Ingeniería Matemática e Informática, Universidad Pública de Navarra and INAMAT, Pamplona ( $\{j$ l. lopez\} @unavarra.es).
} 
The three first canonical integrals, the Airy function, the Pearcey integral, and the swallowtail integral, are the most important ones in applications. The asymptotic behavior of the Airy function is well-known; the asymptotics of the Pearcey integral has been considered in recent works $[9,10]$ and in other more classical works $[7,14,15]$. In this paper we focus our attention on the swallowtail integral, for which a complete asymptotic analysis is not known.

In [6] we have investigated the asymptotic behavior of $\Psi(x, y, z)$ for large $|x|$ and fixed $y$ and $z$. In this work we derive new asymptotic expansions that provide satisfactory approximations of $\Psi(x, y, z)$ for large $|y|$ and moderate values of $x$ and $z$. The analysis of the case $|z|$ large and fixed $x$ and $y$ deserves a separate analysis in a forthcoming paper (see the last paragraph of Section 4 for further details). Once these three cases are fully investigated, the asymptotic analysis of the swallowtail integral will be complete and reference [1, Sec. 36.11], dedicated to the asymptotic analysis of the catastrophe integrals, may be updated. Moreover, the asymptotic analysis of these three cases would lead to a complete description of the dynamics of the optical swallowtail catastrophe when either of the two transverse spatial variables is large or the control parameter is large [19].

The analysis here is different from the analysis in [6] as the location of the saddle points and of the steepest descent paths is different. As a consequence, the complex $y$-plane is divided into asymptotic regions different from those found in [6] for the $x$-plane, and the Stokes lines are also different.

In the following section, we analyze the saddle point features of the swallowtail integral for large $|y|$ and fixed $x$ and $z$. In Section 3 we use a simplification of the saddle point method proposed in [11] to derive complete asymptotic expansions of $\Psi(x, y, z)$ for large $|y|$. Section 4 contains a summary of the discussion and some numerical experiments. Throughout the paper we use the principal argument $\arg w \in(-\pi, \pi]$ for any complex number $w$.

2. Preliminaries. The integral (1.1) converges for $0<\arg y<\pi$ and real $x$, or for real $x, y$, and $z$, but the integrand is highly oscillatory, and thus it is not an appropriate starting point for numerical or analytical approximations. We can find a different representation of the swallowtail integral with a monotonic integrand that is more appropriate for designing approximations. We derive it in the following form: we split the integration interval $(-\infty, \infty)$ at $t=0$ and rotate the path $(-\infty, 0)$ by an angle $-\frac{\pi}{10}$ and the path $(0, \infty)$ by an angle $\frac{\pi}{10}$. After these manipulations, the swallowtail integral may be written in the form

$$
\begin{aligned}
\Psi(x, y, z)=e^{-i \frac{\pi}{10}} & S\left(x e^{-4 i \frac{\pi}{5}}, y e^{3 i \frac{\pi}{10}}, z e^{-3 i \frac{\pi}{5}}\right) \\
& +e^{i \frac{\pi}{10}} S\left(x e^{4 i \frac{\pi}{5}}, y e^{7 i \frac{\pi}{10}}, z e^{3 i \frac{\pi}{5}}\right)
\end{aligned}
$$

with

$$
S(x, y, z):=\int_{0}^{\infty} e^{-u^{5}+x u^{3}+y u^{2}+z u} d u
$$

The integral $S(x, y, z)$ is absolutely convergent for all complex values of $x, y$, and $z$ and defines an entire function of the three variables. Therefore, the right-hand side of (2.1) and (2.2) constitute an explicit representation of the analytic continuation of the swallowtail integral $\Psi(x, y, z)$ to all complex values of $x, y$, and $z$. Thus, it is more convenient to work with the representation (2.1)-(2.2) of the swallowtail integral. The derivation of the above representation of the swallowtail integral is based on a similar one given in [14] for the Pearcey integral. 


\section{The saddle point analysis of the integral $S(x, y, z)$.}

3.1. Saddle points and steepest descent paths of $\boldsymbol{S}(\boldsymbol{x}, \boldsymbol{y}, \boldsymbol{z})$. Define $\theta:=\arg y$. After the change of variable $u=t\left(\frac{2|y|}{5}\right)^{1 / 3}$ in the integral (2.2), we find that it may be written in the form

$$
S(x, y, z)=\left(\frac{2|y|}{5}\right)^{1 / 3} \int_{0}^{\infty} e^{|y|\left(\frac{2|y|}{5}\right)^{2 / 3} f(t)+\frac{2|y|}{5} x t^{3}+z\left(\frac{2|y|}{5}\right)^{1 / 3} t} d t
$$

where the phase function is $f(t):=e^{i \theta} t^{2}-\frac{2}{5} t^{5}$. This phase function has four saddle points: $t_{0}:=0$ and $\bar{t}_{k}:=e^{i \frac{\theta+2 k \pi}{3}}, k=0,1,-1$. From the steepest descent method [18, Chap. 2], or its simplified modification [11], we know that the asymptotically relevant saddle points are those for which the integration path $C:=[0, \infty)$ in (3.1) can be deformed into a new path $\Gamma$ containing portions of steepest descent paths that include all the relevant saddle points. Therefore, the points $\bar{t}_{1}$ and $\bar{t}_{-1}$ do not have any influence in the analysis. Thus, we only consider the saddle points $t_{0}=0$ and $\bar{t}_{0}=e^{i \frac{\theta}{3}}$ (see Figure 3.1).

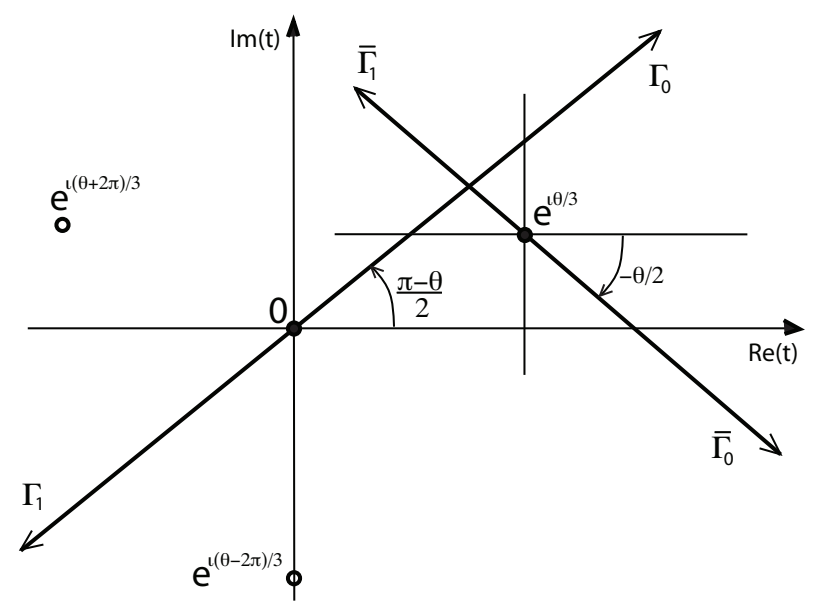

FIG. 3.1. Saddle points $t_{0}:=0$ and $\bar{t}_{k}:=e^{i \frac{\theta+2 k \pi}{3}}, k=0,1,-1$, of the phase function in (3.1) and the steepest descent paths at $t_{0}=0$ and at $\bar{t}_{0}=e^{i \frac{\theta}{3}}$. The integration path $[0, \infty)$ in (3.1) can be deformed either: (i) to a portion of one of the steepest descent paths $\Gamma_{0}$ or $\Gamma_{1}$ at the point $t_{0}=0$ or (ii) to the union of a portion of one of the steepest descent paths $\Gamma_{0}$ or $\Gamma_{1}$ at $t_{0}=0$ and of another portion of the steepest descent path $\bar{\Gamma}_{0} \cup \bar{\Gamma}_{1}$ at $\bar{t}_{0}=e^{i \frac{\theta}{3}}$. Therefore, the saddle points $\bar{t}_{ \pm 1}=e^{i \frac{\theta \pm 2 \pi}{3}}$ do not have any influence in the asymptotic analysis of (3.1). This picture corresponds to a certain $\theta>0$. For negative $\theta$, the picture is symmetric with respect to the axis $\operatorname{Re}(t)$, interchanging also $\Gamma_{0}$ and $\Gamma_{1}$.

The application of the standard steepest descent method to the integral $S(x, y, z)$ is not straightforward as the steepest descent paths of $f(t)$ at the saddle points are difficult to handle. But we know from [11] that, instead of the steepest descent paths of the phase function $f(t)$, we may consider the steepest descent paths of the "main part" $f_{m}(t)$ of $f(t)$ at the relevant saddle points. The "main part" $f_{m}(t)$ is just the Taylor polynomial of degree $m$ of $f(t)$ at the saddle point $T: f_{m}(t):=f(T)+f^{(m)}(T)(t-T)^{m} / m !$, where $m$ is the order of the first nonvanishing derivative of $f(t)$ at $t=T\left(f^{(m)}(T) \neq 0\right.$ and $f^{(k)}(T)=0$ for $k=1,2,3, \ldots, m-1)$. The steepest descent paths of $f_{m}(t)$ are just straight lines [11]. 
Then, the first point of the asymptotic analysis of (3.1) is the computation, at each relevant saddle point $t_{0}$ and $\bar{t}_{0}$, of the steepest descent paths of $f_{m}(t)$. Following [11] (see [6] for a similar derivation), we find that they are the following ones:

- At the saddle point $T=t_{0}=0$ we have that $m=2, \phi=\theta, f_{2}(t)=e^{i \theta} t^{2}$, and its steepest descent paths are the following two half-lines (see Figure 3.1):

$$
\Gamma_{k}:=\left\{r e^{i \theta_{k}} ; \quad \theta_{k}:=\frac{(1-2 k) \pi-\theta}{2} ; r \geq 0\right\}, \quad k=0,1 .
$$

- At the saddle point $T=\bar{t}_{0}:=e^{i \frac{\theta}{3}}$ we have that $m=2, \phi=\theta+\pi$, $f_{2}(t)=\frac{3}{5} e^{5 i \frac{\theta}{3}}-3 e^{i \theta}\left(t-e^{i \frac{\theta}{3}}\right)^{2}$, and its steepest descent paths are the following two half-lines (see Figure 3.1):

$$
\bar{\Gamma}_{k}:=\left\{e^{i \frac{\theta}{3}}+r e^{i \bar{\theta}_{k}} ; \quad \bar{\theta}_{k}:=\frac{2 k \pi-\theta}{2} ; r \geq 0\right\}, \quad k=0,1 .
$$

3.2. Deformation of the integration path. The second part of our analysis consists in showing that the original path of integration $[0, \infty)$ in (3.1) may be deformed to another path $\Gamma$ that is more appropriate for the asymptotic analysis, and hence we write:

$$
S(x, y, z)=\left(\frac{2|y|}{5}\right)^{1 / 3} \int_{\Gamma} e^{|y|\left(\frac{2|y|}{5}\right)^{2 / 3} f(t)+\frac{2|y|}{5} x t^{3}+z\left(\frac{2|y|}{5}\right)^{1 / 3} t} d t .
$$

In this formula, $\Gamma:=\Gamma_{s} \cup \Gamma_{\epsilon}$, where $\Gamma_{s}$ is the union of one portion of one of the two half-lines $\Gamma_{0}$ or $\Gamma_{1}$ and a portion of $\bar{\Gamma}_{0} \cup \bar{\Gamma}_{1}$ in such a way that it contains the relevant saddle point(s) $t_{0}$ and/or $\bar{t}_{0}$. The "irrelevant" path $\Gamma_{\epsilon}$ is necessary to complete the deformation of $[0, \infty)$, but the integral over this path is exponentially small compared with the integral over the path $\Gamma_{s}$. The analysis of the deformation depends on the angle $\theta$ (see Figures 3.2, 3.3, and 3.4) and all the deformations used in the following four paragraphs are justified by the use of Cauchy's residue theorem:

- When $\frac{4 \pi}{5}<\theta \leq \pi$ we have that $\left|\theta_{0}\right|=\left|\frac{\pi-\theta}{2}\right|<\frac{\pi}{10}$. We can deform the path $[0, \infty)$ to the path $\Gamma_{0}$, whose end point is the saddle point $t_{0}=0$. We have that $\Gamma_{s}=\Gamma_{0}, \Gamma_{\epsilon}$ is empty, and $\Gamma_{s}=\Gamma_{0}$ (see Figure 3.2).

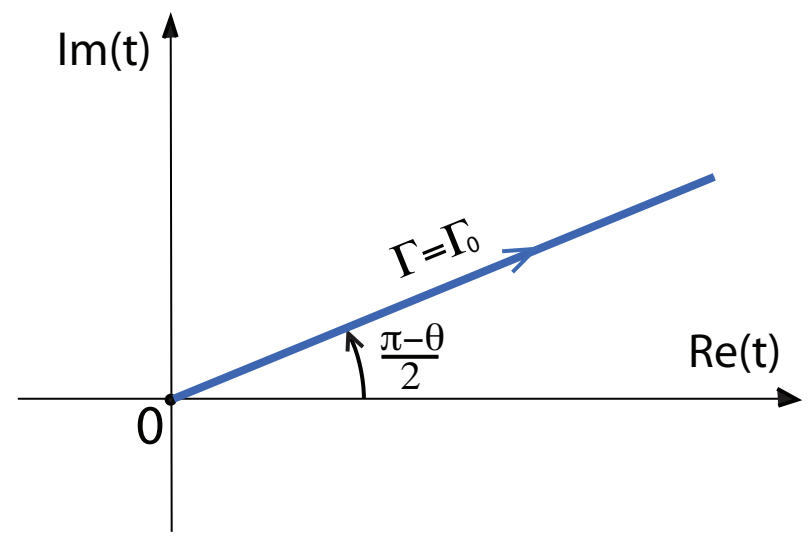

FIG. 3.2. When $\frac{4 \pi}{5}<\theta \leq \pi$, the path $[0, \infty)$ is deformed to the path $\Gamma=\Gamma_{0}$. 
- When $\frac{3 \pi}{5} \leq \theta \leq \frac{4 \pi}{5}$ we can deform the path $[0, \infty)$ to the path $\Gamma=\Gamma_{s} \cup \Gamma_{\epsilon}$, where $\Gamma_{s}$ is the segment $\overline{0 A}$ of $\Gamma_{0}$ plus the segment $\overline{A B}$ of $\bar{\Gamma}_{0}$ (see Figure 3.3a for $\frac{3 \pi}{5}<\theta<\frac{4 \pi}{5}$ and Figure $3.3 \mathrm{~b}$ for $\left.\theta=\frac{3 \pi}{5}\right) . \Gamma_{\epsilon}$ is the interval $[B, \infty)$.

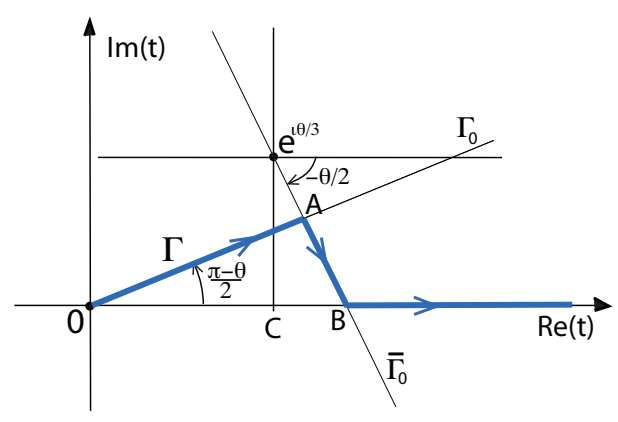

(a)

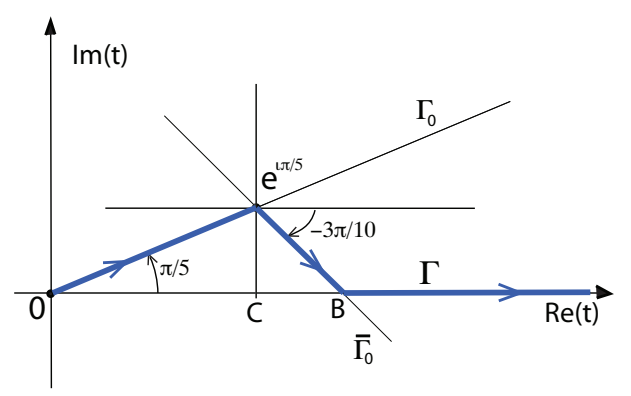

(b)

FIG. 3.3. When $\frac{3 \pi}{5} \leq \theta \leq \frac{4 \pi}{5}$ we can deform the path $[0, \infty)$ to the path $\Gamma=\overline{O A} \cup \overline{A B} \cup[B, \infty)$ represented in (a) for $\frac{3 \pi}{5}<\theta \leq \frac{4 \pi}{5}$ or in (b) for $\theta=\frac{3 \pi}{5}$ (when $\theta=\frac{3 \pi}{5}$, then $A=t_{0}=e^{i \frac{\theta}{3}}=e^{i \frac{\pi}{5}}$ ). In these figures $A:=\Gamma_{0} \cap \bar{\Gamma}_{0}$ and $B:=\bar{\Gamma}_{0} \cap \operatorname{Re}(t)$-axis.

- When $0 \leq \theta<\frac{3 \pi}{5}$ we can deform the path $[0, \infty)$ to the path $\Gamma=\Gamma_{s} \cup \Gamma_{\epsilon}$, where $\Gamma_{s}$ is the segment $\overline{0 A}$ of $\Gamma_{0}$ plus the segment $\overline{A B}$ of $\bar{\Gamma}_{0} \cup \bar{\Gamma}_{1}$ (see Figure 3.4). $\Gamma_{\epsilon}$ is the interval $[B, \infty)$.

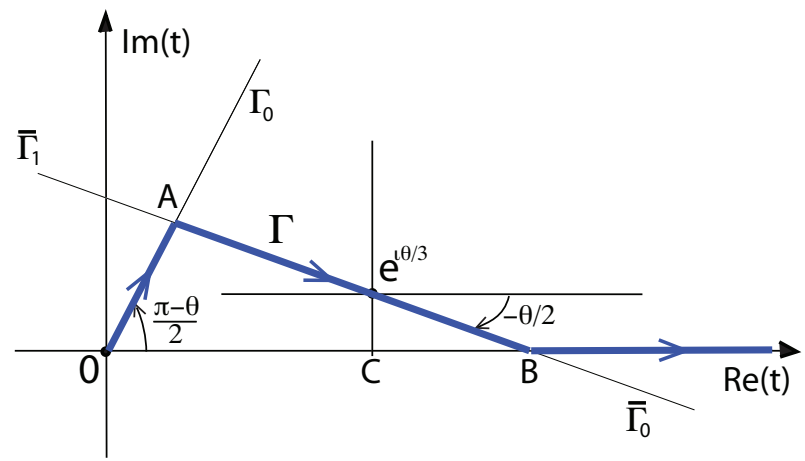

FIG. 3.4. When $0 \leq \theta<\frac{3 \pi}{5}$, the path $[0, \infty)$ can be deformed to the path $\Gamma=\overline{O A} \cup \overline{A B} \cup[B, \infty)$. In this figure $A:=\Gamma_{0} \cap \bar{\Gamma}_{1}$ and $B:=\bar{\Gamma}_{0} \cap \operatorname{Re}(t)$-axis.

- For negative $\theta$ the analysis is similar: (i) for $-\pi<\theta<-\frac{4 \pi}{5}$ the analysis is identical to the case $\frac{4 \pi}{5}<\theta \leq \pi$; (ii) for $-\frac{4 \pi}{5} \leq \theta \leq-\frac{3 \pi}{5}$ it is identical to the case $\frac{3 \pi}{5} \leq \theta \leq \frac{4 \pi}{5}$; (iii) for $-\frac{3 \pi}{5}<\theta \leq 0$ it is identical to the case $0 \leq \theta<\frac{3 \pi}{5}$. The only difference is that the figures are symmetric with respect to the $\operatorname{Re}(t)$-axis and $\bar{\Gamma}_{0}$ is replaced by $\bar{\Gamma}_{1}$.

3.3. Computation of the integrals over the steepest descent paths. The third point of our analysis is the computation of the right-hand side of (3.2) when $\Gamma$ is one of the paths described in the previous section. We only give details for $\theta \geq 0$ as the case $\theta \leq 0$ is symmetric. The following two observations are essential in the analysis: 
- Regardless of the value of $\theta$, the segment $\overline{O A}$ is present ${ }^{1}$ in $\Gamma_{s}$. Besides, when $0 \leq \theta \leq \frac{4 \pi}{5}$, the segment $\overline{A B}$ is also present in $\Gamma_{s}$, although only for the argument $0 \leq \theta \leq \frac{3 \pi}{5}$, the saddle point $\bar{t}_{0}=e^{i \frac{\theta}{3}}$ is in $\overline{A B}$. On the other hand, among the two saddle points $t_{0}=0$ and $\bar{t}_{0}=e^{i \frac{\pi}{3}}$, the most relevant one is the one for which $\Re[f(t)]$ is maximal, and this depends on $\theta: \Re[f(0)]=0$ and $\Re\left[f\left(e^{i \frac{\theta}{3}}\right)\right]=\frac{3}{5} \cos \left(\frac{5 \theta}{3}\right)$. Therefore, $t_{0}=0$ is the relevant saddle point for $|\theta|>\frac{3 \pi}{10}, \bar{t}_{0}=e^{i \frac{\theta}{3}}$ is the relevant saddle point for $|\theta|<\frac{3 \pi}{10}$, and both are equally relevant for $\theta=\frac{3 \pi}{10}$.

- For $\frac{\pi}{2} \leq \theta \leq \pi$ we have that $\Re[f(t)]$ is a decreasing function in $[0, \infty)$ and $\Re[f(t)] \leq \Re[f(0)]=0$ for $t \in[0, \infty)$. For $0 \leq \theta<\frac{\pi}{2}$ we have that the maximum of $\Re[f(t)]$ is located at $t=(\cos \theta)^{1 / 3}$ and is a decreasing function in $\left[(\cos \theta)^{1 / 3}, \infty\right)$. But $B \geq C:=\cos \left(\frac{\theta}{3}\right) \geq(\cos \theta)^{1 / 3}$, and then $\Re[f(t)]$ is a decreasing function in $\Gamma_{\epsilon}=[B, \infty)$. In any steepest descent path, $\Re[f(t)]$ decreases as $t$ runs away from the saddle point, and then $\Re[f(t)] \leq \Re[f(B)]<\Re\left[f\left(e^{i \frac{\theta}{3}}\right)\right]$ for $t \in \Gamma_{\epsilon}=[B, \infty)$.

From the above first observation we conclude that we may consider that $\Gamma_{s}=\overline{O A}$ for $\frac{3 \pi}{10}<|\theta| \leq \pi$ and $\Gamma_{s}=\overline{O A} \cup \overline{A B}$ for $0 \leq|\theta|<\frac{3 \pi}{5}$. From the second observation we conclude that the contribution of $\Gamma_{\epsilon}$ is exponentially small compared to the contribution of $\Gamma_{s}$ for any $\theta$. In summary,

$$
S(x, y, z) \sim \begin{cases}S_{0}(x, y, z) & \text { if } \frac{3 \pi}{10}<|\theta| \leq \pi, \\ S_{0}(x, y, z)+\bar{S}(x, y, z) & \text { if }|\theta|<\frac{3 \pi}{5},\end{cases}
$$

where

$$
S_{0}(x, y, z):=\left(\frac{2|y|}{5}\right)^{1 / 3} \int_{0}^{A} e^{|y|\left(\frac{2|y|}{5}\right)^{2 / 3} f_{2}(t)} e^{|y|\left(\frac{2|y|}{5}\right)^{2 / 3}\left[f(t)-f_{2}(t)\right]+\frac{2|y|}{5} x t^{3}+z\left(\frac{2|y|}{5}\right)^{1 / 3} t} d t
$$

and

$$
\bar{S}(x, y, z):=\left(\frac{2|y|}{5}\right)^{1 / 3} \int_{A}^{B} e^{|y|\left(\frac{2|y|}{5}\right)^{2 / 3} f_{2}(t)} e^{|y|\left(\frac{2|y|}{5}\right)^{2 / 3}\left[f(t)-f_{2}(t)\right]+\frac{2|y|}{5} x t^{3}+z\left(\frac{2|y|}{5}\right)^{1 / 3} t} d t .
$$

In (3.4) we have $f_{2}(t)=e^{i \theta} t^{2}$ and in (3.5), $f_{2}(t)=\frac{3}{5} e^{5 i \frac{\theta}{3}}-3 e^{i \theta}\left(t-e^{i \frac{\theta}{3}}\right)^{2}$. Figure 3.5a displays the different asymptotic behavior of $S(x, y, z)$ in the complex $y$-plane according to (3.3).

In the red sector $|\theta|<\frac{3 \pi}{10}, S(x, y, z) \sim S_{0}(x, y, z)+\bar{S}(x, y, z)$, whereas in the blue sector $\frac{3 \pi}{5}<|\theta| \leq \pi, S(x, y, z) \sim S_{0}(x, y, z)$. In the purple sector $\frac{3 \pi}{10}<|\theta| \leq \frac{3 \pi}{5}$, either of the two approximations is valid as $\bar{S}(x, y, z)$ is exponentially small compared with $S_{0}(x, y, z)$. Inside the red sector $|\theta|<\frac{3 \pi}{10}, \bar{S}(x, y, z)$ dominates ${ }^{2} S_{0}(x, y, z)$. The Stokes lines are the lines $\arg (y)= \pm \frac{3 \pi}{10}$, where both, $S_{0}(x, y, z)$ and $\bar{S}(x, y, z)$, are of the same order.

3.4. The approximate computation of $S_{0}(x, y, z)$ and $\bar{S}(x, y, z)$. The fourth point of the analysis is the approximation of the integrals $S_{0}(x, y, z)$ and $\bar{S}(x, y, z)$. Again, we only give details for positive $\theta$ as the analysis for negative $\theta$ is identical. After the change of variable $t \rightarrow\left(\frac{5}{2}\right)^{\frac{1}{3}} e^{i \frac{\pi-\theta}{2}}|y|^{-5 / 6} t$ in (3.4), we find

$$
S_{0}(x, y, z)=\frac{1}{(-y)^{1 / 2}} \int_{0}^{A_{y}} e^{-t^{2}} g(x, y, z, t) d t,
$$

\footnotetext{
${ }^{1}$ Although when $\theta=0$ this segment is irrelevant because $A=0$.

${ }^{2}$ Moreover, when $\theta=0, A=0$ and thus $S_{0}(x, y, z)=0$.
} 


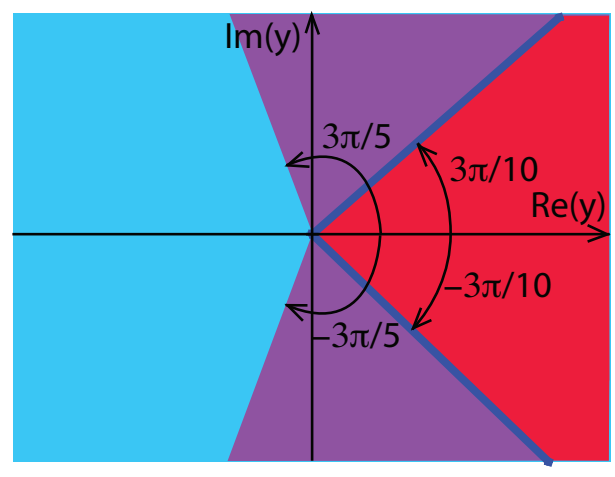

(a)

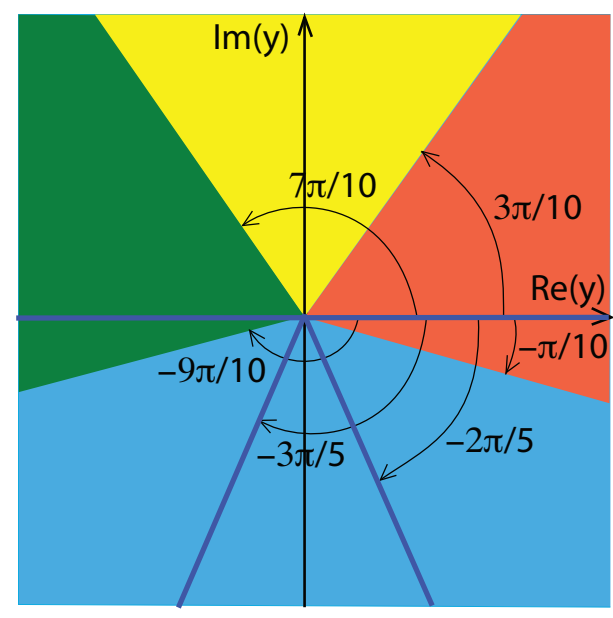

(b)

FIG. 3.5. (a) The saddle point analysis of the integral (2.2) is different in the two regions of the complex $y$-plane separated by the lines $\arg y= \pm \frac{3 \pi}{10} ;(b)$ both, $S_{0}\left(x e^{-4 i \frac{\pi}{5}}, y e^{3 i \frac{\pi}{10}}, z e^{-3 i \frac{\pi}{5}}\right)$ and $S_{0}\left(x e^{4 i \frac{\pi}{5}}, y e^{7 i \frac{\pi}{10}}, z e^{3 i \frac{\pi}{5}}\right)$ contribute to the asymptotic behavior of the swallowtail integral in the whole complex $y$-plane (except for $\arg y=-\frac{3 \pi}{10}$ and $\arg y=-\frac{7 \pi}{10}$ when they respectively vanish). But $\bar{S}\left(x e^{-4 i \frac{\pi}{5}}, y e^{3 i \frac{\pi}{10}}, z e^{-3 i \frac{\pi}{5}}\right)$ and $\bar{S}\left(x e^{4 i \frac{\pi}{5}}, y e^{7 i \frac{\pi}{10}}, z e^{3 i \frac{\pi}{5}}\right)$ contribute differently in the four sectors depicted in the figure: both of them contribute in the blue sector $-\frac{9 \pi}{10} \leq \arg y \leq-\frac{\pi}{10}$, none in the yellow sector $\frac{3 \pi}{10}<\arg y<\frac{7 \pi}{10}$, only $\bar{S}\left(x e^{-4 i \frac{\pi}{5}}, y e^{3 i \frac{\pi}{10}}, z e^{-3 i \frac{\pi}{5}}\right)$ in the red sector $-\frac{\pi}{10}<\arg y \leq \frac{3 \pi}{10}$, and only $\bar{S}\left(x e^{4 i \frac{\pi}{5}}, y e^{7 i \frac{\pi}{10}}, z e^{3 i \frac{\pi}{5}}\right)$ in the green sector $-\pi \leq \theta<-\frac{9 \pi}{10}$ and $\frac{7 \pi}{10} \leq \arg y \leq \pi$. The Stokes lines are the thick blue lines $\arg y=-\frac{3 \pi}{5}$, $-\frac{2 \pi}{5}, 0$, and $\pi$.

with $A_{y}:=|A|\left(\frac{2}{5}\right)^{1 / 3}\left|y^{5 / 6}\right| \geq 0$ and

$$
g(x, y, z, t):=\exp \left\{-\frac{t^{5}}{(-y)^{5 / 2}}+\frac{x t^{3}}{(-y)^{3 / 2}}+\frac{z t}{(-y)^{1 / 2}}\right\} .
$$

From here, the computation of the asymptotic expansion of this integral follows from Watson's Lemma [18, Chap. 1, Sec. 5], [11]. Replacing $g(x, y, z, t)$ by its Taylor expansion at $t=0$, interchanging sum and integral, and neglecting exponentially small terms, we find that $S_{0}(x, y, z)=0$ for $\theta=0$, and, for $\theta \neq 0$,

$$
S_{0}(x, y, z) \sim \frac{1}{2} \sum_{n=0}^{\infty} \Gamma\left(\frac{n+1}{2}\right) \frac{B_{n}(x, z)}{(-y)^{(n+1) / 2}} \quad \text { as } \quad|y| \rightarrow \infty
$$

with

$$
B_{n}(x, z):=\sum_{5 k+3 j+l=n} \frac{(-1)^{k} x^{j} z^{l}}{k ! j ! l !}
$$

From the differential equation $g^{\prime}(x,-1, z, t)=\left(z+3 x t^{2}-5 t^{4}\right) g(x,-1, z, t)$ in the variable $t$, it is straightforward to see that the coefficients $B_{n}(x, z)$ satisfy the recurrence relation

$$
B_{n}(x, z)=\frac{z B_{n-1}(x, z)+3 x B_{n-3}(x, z)-5 B_{n-5}(x, z)}{n},
$$


with

$$
\begin{array}{lll}
B_{0}(x, z)=1, & B_{1}(x, z)=z, & B_{2}(x, z)=\frac{z^{2}}{2}, \\
B_{3}(x, z)=x+\frac{z^{3}}{6}, & B_{4}(x, z)=x z+\frac{z^{4}}{24} . &
\end{array}
$$

On the other hand, after the change of variables $t \rightarrow u$ defined by

$$
t=e^{i \frac{\theta}{3}}+e^{-i \frac{\theta}{2}} \frac{\left(\frac{5}{2}\right)^{1 / 3}}{|y|^{5 / 6} \sqrt{3}} u
$$

in the integral (3.5), we find

$$
\bar{S}(x, y, z)=\frac{1}{\sqrt{3 y}} e^{h(x, y, z)} \int_{A_{y}}^{B_{y}} e^{-u^{2}+u w(x, y, z)} g(x, y, z, u) d u,
$$

with

$$
\begin{gathered}
A_{y}:=\left(\frac{2}{5}\right)^{1 / 3} \sqrt{3} e^{i \frac{\theta}{2}}\left(A-e^{i \frac{\theta}{3}}\right)|y|^{5 / 6}<0, \\
B_{y}:=\left(\frac{2}{5}\right)^{1 / 3} \sqrt{3} e^{i \frac{\theta}{2}}\left(B-e^{i \frac{\theta}{3}}\right)|y|^{5 / 6}>0 \\
h(x, y, z):=\frac{3}{5}\left(\frac{2}{5}\right)^{2 / 3} y^{5 / 3}+\frac{2 x y}{5}+z\left(\frac{2 y}{5}\right)^{1 / 3}, \\
w(x, y, z):=\sqrt{3}\left(\frac{2}{5}\right)^{2 / 3} x y^{1 / 6}+\frac{z}{\sqrt{3 y}}, \\
g(x, y, z, u)=\exp \left\{\frac{a_{2}(x, y, z)}{y^{5 / 9}} u^{2}+\frac{a_{3}(x, y, z)}{y^{5 / 6}} u^{3}+\frac{a_{4}(x, y, z)}{y^{10 / 9}} u^{4}+\frac{a_{5}(x, y, z)}{y^{25 / 18}} u^{5}\right\},
\end{gathered}
$$

and

$$
\begin{array}{ll}
a_{2}(x, y, z):=\frac{\left(\frac{2}{5}\right)^{1 / 3} x}{y^{1 / 9}}, & a_{3}(x, y, z):=\frac{1}{3 \sqrt{3}}\left[-2^{5 / 3} 5^{1 / 3}+\frac{x}{y^{2 / 3}}\right], \\
a_{4}(x, y, z):=-\frac{2^{1 / 3} 5^{2 / 3}}{9 y^{5 / 9}}, & a_{5}(x, y, z):=-\frac{1}{3^{5 / 2} y^{10 / 9}} .
\end{array}
$$

Replacing the Taylor expansion of $g(x, y, z, u)$ at $u=0$ in (3.7), interchanging sum and integral, and neglecting exponentially small terms, we find that

$$
\bar{S}(x, y, z) \sim \frac{1}{\sqrt{3 y}} e^{h(x, y, z)+w^{2}(x, y, z) / 4} \sum_{n=0}^{\infty} \frac{C_{n}(x, y, z) A_{n}(x, y, z)}{y^{5 n / 18}},
$$

where, for $n=0,1,2,3, \ldots, C_{n}(x, y, z)$ are the Taylor coefficients of $g\left(x, y, z, y^{5 / 18} u\right)$ at $u=0$ :

$$
C_{n}(x, y, z):=\sum_{2 n_{2}+3 n_{3}+4 n_{4}+5 n_{5}=n} \frac{a_{2}^{n_{2}}(x, y, z) a_{3}^{n_{3}}(x, y, z) a_{4}^{n_{4}}(x, y, z) a_{5}^{n_{5}}(x, y, z)}{n_{2} ! n_{3} ! n_{4} ! n_{5} !} .
$$


It is obvious that $C_{0}(x, y, z)=1$ and $C_{1}(x, y, z)=0$. And, from the following differential equation with respect to the variable $u$,

$$
g^{\prime}\left(x, y, z, y^{5 / 18} u\right)=g\left(x, y, z, y^{5 / 18} u\right) \sum_{k=2}^{5} k a_{k}(x, y, z) u^{k-1}
$$

it is straightforward to see that, for $n=2,3,4, \ldots$, the coefficients $C_{n}(x, y, z)$ satisfy the recurrence relation

$$
C_{n}(x, y, z)=\frac{1}{n} \sum_{k=2}^{\min \{n, 5\}} k a_{k}(x, y, z) C_{n-k}(x, y, z) .
$$

On the other hand, the functions $A_{n}(x, y, z)$ are the integrals

$$
\begin{aligned}
A_{n}(x, y, z) & :=\int_{-\infty}^{\infty} u^{n} e^{-(u-w(x, y, z) / 2)^{2}} d u=\int_{-\infty}^{\infty}\left[t+\frac{w(x, y, z)}{2}\right]^{n} e^{-t^{2}} d t \\
& =\sum_{k=0}^{n}\left(\begin{array}{c}
n \\
k
\end{array}\right)\left(\frac{w(x, y, z)}{2}\right)^{n-k} \int_{-\infty}^{\infty} t^{k} e^{-t^{2}} d t \\
& =\sum_{k=0}^{\lfloor n / 2\rfloor}\left(\begin{array}{c}
n \\
2 k
\end{array}\right)\left(\frac{w(x, y, z)}{2}\right)^{n-2 k} \Gamma\left(k+\frac{1}{2}\right) .
\end{aligned}
$$

The two first functions $A_{n}(x, y, z)$ are

$$
A_{0}(x, y, z)=\sqrt{\pi}, \quad A_{1}(x, y, z)=\frac{\sqrt{\pi}}{2} w(x, y, z)
$$

and, for $n=2,3,4, \ldots$, the remaining ones may be computed from the recurrence relation

$$
A_{n}(x, y, z)=\frac{w(x, y, z)}{2} A_{n-1}(x, y, z)+\frac{n-1}{2} A_{n-2}(x, y, z) .
$$

This recurrence relation follows straightforwardly by integrating by parts in the integral definition of $A_{n}(x, y, z)$.

On the one hand, from (3.8) and (3.10) it is obvious that the coefficients satisfy $C_{n}=\mathcal{O}(1)$ when $|y| \rightarrow \infty$ with fixed $x$ and $z$. From (3.11) it is straightforward to see that $A_{n}(x, y, z)=\mathcal{O}\left(w^{n}(x, y, z)\right)=\mathcal{O}\left(y^{n / 6}\right)$. Thus, every term of the asymptotic expansion of $\bar{S}(x, y, z)$ in (3.9) is of the order $\mathcal{O}\left(y^{-n / 9}\right)$ as $|y| \rightarrow \infty$ for bounded $x$ and $z$.

4. Summary of the discussion and numerical experiments. From (3.3), (3.6), and (3.9) we see that, when $|y| \rightarrow \infty, S(x, y, z)$ is of the order $\mathcal{O}\left(y^{-1 / 2}\right)$ if $|\arg y|>\frac{3 \pi}{10}$ and of the order $\mathcal{O}\left(y^{-1 / 2} e^{h(x, y, z)+w^{2}(x, y, z) / 4}\right)$ if $|\arg y| \leq \frac{3 \pi}{10}$. Thus, $S(x, y, z)$ has an exponential behavior in the red region of Figure 3.5a and a power behavior in the blue and purple regions. The Stokes lines for $S(x, y, z)$ are the rays $\arg y= \pm \frac{3 \pi}{10}$.

In order to determine the asymptotic behavior of the swallowtail integral $\Psi(x, y, z)$, we must use the relations (2.1) and (3.3). The first function $S(x, y, z)$ on the right-hand side of (2.1) is evaluated at $y e^{3 i \frac{\pi}{10}}$ and the second one at $y e^{7 i \frac{\pi}{10}}$. Therefore, the complex $y$-plane is divided into the four regions depicted in Figure 3.5b, according to the asymptotic behavior of 
$\Psi(x, y, z):$

$$
\begin{aligned}
\Psi(x, y, z) \sim & e^{-i \frac{\pi}{10}} S_{0}\left(x e^{-4 i \frac{\pi}{5}}, y e^{3 i \frac{\pi}{10}}, z e^{-3 i \frac{\pi}{5}}\right) \\
& +e^{i \frac{\pi}{10}} S_{0}\left(x e^{4 i \frac{\pi}{5}}, y e^{7 i \frac{\pi}{10}}, z e^{3 i \frac{\pi}{5}}\right) \\
& +e^{-i \frac{\pi}{10}} \bar{S}\left(x e^{-4 i \frac{\pi}{5}}, y e^{3 i \frac{\pi}{10}}, z e^{-3 i \frac{\pi}{5}}\right) \chi_{\left[0, \frac{3 \pi}{5}\right)}\left(\left|\arg y+\frac{3 \pi}{10}\right|\right) \\
& +e^{i \frac{\pi}{10}} \bar{S}\left(x e^{4 i \frac{\pi}{5}}, y e^{7 i \frac{\pi}{10}}, z e^{3 i \frac{\pi}{5}}\right) \chi_{\left[0, \frac{3 \pi}{5}\right)}\left(\left|\arg y+\frac{7 \pi}{10}\right|\right),
\end{aligned}
$$

where $\chi_{[a, b)}(\theta)$ is the characteristic function of the interval $[a, b)$. The function $S_{0}(x, y, z)$ is approximated by the right-hand side of (3.6) and $\bar{S}(x, y, z)$ by the right-hand side of (3.9). The Stokes lines of the swallowtail integral $\operatorname{are} \arg y=-\frac{3 \pi}{5},-\frac{2 \pi}{5}, 0$, and $\pi$, and separate the complex $y$-plane into four different sectors:

$$
\begin{aligned}
& -\pi<\arg y<-\frac{3 \pi}{5}, \\
& -\frac{2 \pi}{5}<\arg y<0, \quad \text { and } \quad 0<\arg y<\pi .
\end{aligned}
$$

Table 4.1 displays the accuracy of the approximations summarized above. We evaluate the swallowtail integral $\Psi(x, y, z)$ by using formula (2.1), evaluating the function $S(x, y, z)$ defined in (2.2) with the NIntegrate command of Wolfram Mathematica 10.4 using 16 digits for the numerical evaluation of the integral.

The asymptotic analysis of $\Psi(x, y, z)$ for large $|x|$ or large $|y|$ has been possible through the splitting (2.1) and the asymptotic approximation of the functions $S(x, y, z)$. But the analysis of the asymptotic behavior of $\Psi(x, y, z)$ for large $|z|$ and fixed $x$ and $y$ is more cumbersome. In this case, the point $t=0$ is not a saddle point of the phase function. This fact translates into numerical instabilities in the asymptotic approximation of $\Psi(x, y, z)$ by means of the splitting (2.1) and the asymptotic approximation of the functions $S(x, y, z)$. Therefore, we plan to investigate the large $z$ asymptotics of the swallowtail integral in a separate paper avoiding the mentioned splitting.

Acknowledgments. This research was supported by the Ministerio de Economía y Competitividad (MTM2017-83490-P). The Universidad Pública de Navarra is acknowledged by its financial support.

\section{REFERENCES}

[1] M. V. BERRY AND C. J. Howls, Integrals with coalescing saddles, in NIST Handbook of Mathematical Functions, F. W. J. Olver, D. W. Lozier, R. F. Boisvert, and C. W. Clark, eds., Cambridge University Press, Cambridge, 2010, pp. 775-793.

[2] J. N. L. CONNOR, Practical methods for the uniform asymptotic evaluation of oscillating integrals with several coalescing saddle points, in Asymptotic and Computational Analysis, R. Wong, ed., Lecture Notes in Pure and Appl. Math., 124, Dekker, New York, 1990, pp. 137-173.

[3] J. N. L. CONNOR AND P. R. CURTIS, A method for the numerical evaluation of the oscillatory integrals associated with the cuspoid catastrophes: application to Pearcey's integral and its derivatives, J. Phys. A, 15 (1982), pp. 1179-1190.

[4] J. N. L. CONNOR, P. R. CURTIS, AND D. FARRElly, A differential equation method for the numerical evaluation of the Airy, Pearcey and swallowtail canonical integrals and their derivatives, Molecular Phys., 48 (1983), pp. 1305-1330.

[5] - The uniform asymptotic swallowtail approximation: practical methods for oscillating integrals with four coalescing saddle points, J. Phys. A, 17 (1984), pp. 283-310.

[6] C. Ferreira, J. L. LóPez, AND E. PÉREz Sinusía, The swallowtail integral in the high oscillatory region, Appl. Math. Comput., 339 (2018), pp. 837-845. 


\section{ETNA}

Kent State University and

Johann Radon Institute (RICAM)

TABLE 4.1

Relative errors in the approximation of the integral $\Psi(x, y, z)$ given in (1.1) by using (4.1)-(3.6)-(3.9) with the series (4.1) and (3.6) truncated after $n$ terms. Several values of $y$ in the four regions given in Figure $3.5 b$ are considered. We can observe that the approximation is more accurate away from the Stokes lines.

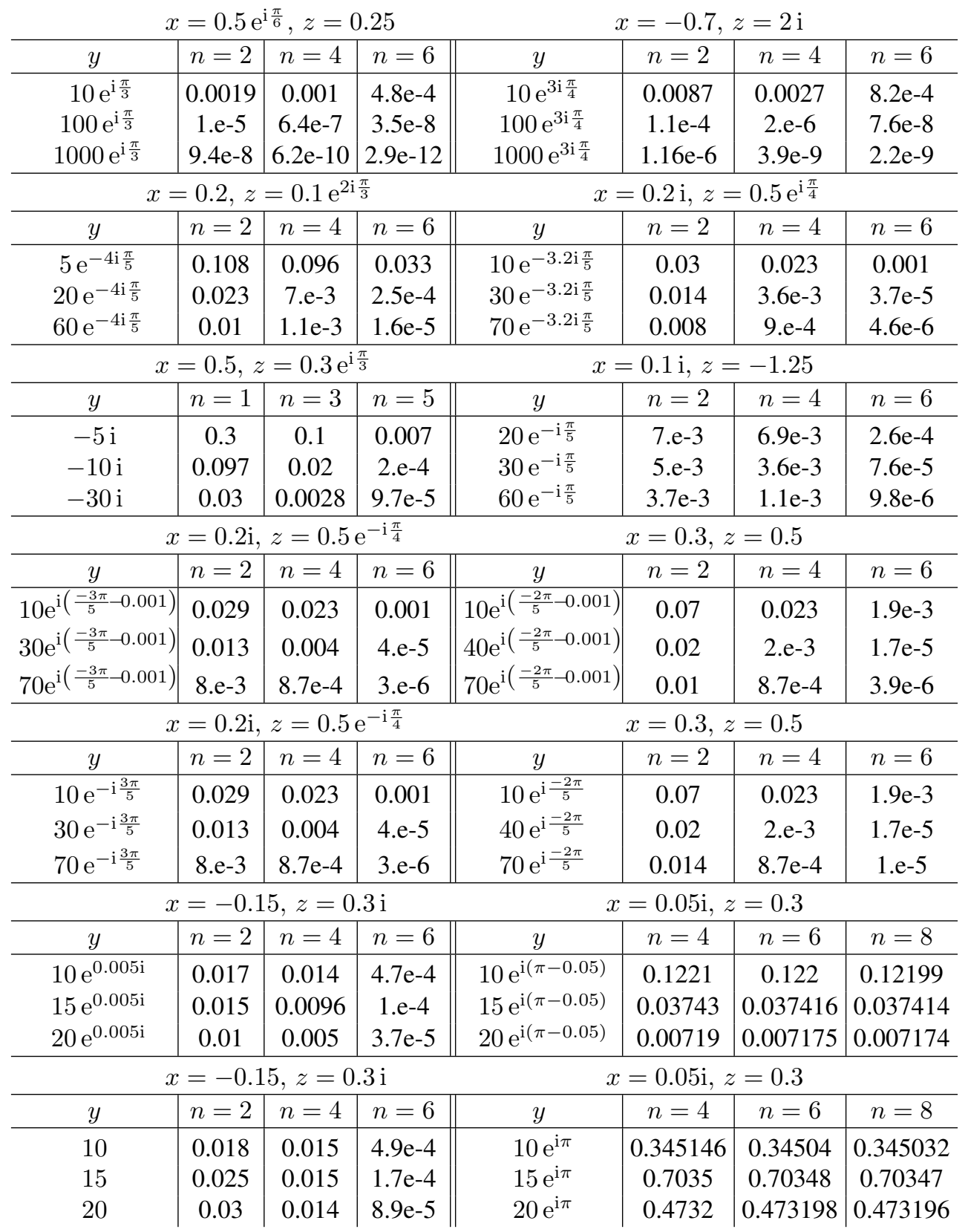

[7] D. Kamins KI, Asymptotic expansion of the Pearcey integral near the caustic, SIAM J. Math. Anal., 20 (1989), pp. 987-1005.

[8] - Asymptotics of the swallowtail integral near the cusp of the caustic, SIAM J. Math. Anal., 23 (1992), pp. 262-285.

[9] J. L. LóPeZ AND P. Pagola, Convergent and asymptotic expansions of the Pearcey integral, J. Math. Anal. Appl., 430 (2015), pp. 181-192. 
ETNA

Kent State University and

Johann Radon Institute (RICAM)

THE SWALLOWTAIL INTEGRAL IN THE HIGHLY OSCILLATORY REGION II

[10] - The Pearcey integral in the highly oscillatory region, Appl. Math. Comput., 275 (2016), pp. 404-410.

[11] J. L. LóPEZ, P. PAgOla AND E. PÉREZ Sinusía, A systematization of the saddle point method. Application to the Airy and Hankel functions, J. Math. Anal. Appl., 354 (2009), pp. 347-359.

[12] Lord Kelvin, Deep water ship-waves, Philos. Mag. (6), 9 (1905), pp. 733-757.

[13] A. B. OLDE DAALHUIS, On the asymptotics for late coefficients in uniform asymptotic expansions of integrals with coalescing saddles, Methods Appl. Anal., 7 (2000), pp. 727-745.

[14] R. B. PARIS, The asymptotic behaviour of Pearcey's integral for complex variables, Proc. Roy. Soc. London Ser. A., 432 (1991), pp. 391-426.

[15] R. B. PARIS AND D. KAMINSKI, Hyperasymptotic evaluation of the Pearcey integral via Hadamard expansions, J. Comput. Appl. Math., 190 (2006), pp. 437-452.

[16] F. URSELL, Integrals with a large parameter: several nearly coincident saddle points, Proc. Cambridge Philos. Soc., 72 (1972), pp. 49-65.

[17] - Ship Hydrodynamics, Water Waves and Asymptotics. Collected works of F. Ursell, 1946-1992. Vol. 2, World Scientific, Singapore, 1994.

[18] R. Wong, Asymptotic Approximations of Integrals, Academic Press, Boston, 1989.

[19] A. Zannotti, F. Diebel, And C. Denz, Dynamics of the optical swallowtail catastrophe, Optica, 4 (2017), pp. $1157-1162$. 